\title{
Contribution de l'analyse en composantes principales à la caractérisation du fer et du chrome contenus dans les effluents de la ville de Nouakchott
}

\author{
Abdoulaye Demba N'DIAYE ${ }^{1 *}$, Mohamed Ould Sid'Ahmed Ould KANKOU ${ }^{2}$ et \\ Baidy $\mathrm{LO}^{1}$ \\ ${ }^{1}$ Laboratoire de Chimie de l'Eau, Institut National de Recherches en Santé Publique de Nouakchott, BP 690, \\ Tél. (222) 52531 75, Mauritanie. \\ ${ }^{2}$ Laboratoire de Chimie de l'Eau et Environnement, Faculté des Sciences et Techniques de l'Université de \\ Nouakchott-BP 5026, Tél. (222) 52513 82, Mauritanie. \\ *Auteur correspondant, E-mail: abdouldemba@yahoo.fr
}

\section{RESUME}

Dans le but de caractériser le fer et le chrome contenus dans les effluents de la ville de Nouakchott par le biais de l'analyse en composantes principales, les campagnes de prélèvements au niveau des effluents de la ville de Nouakchott ont été effectuées hebdomadairement au cours du mois de mars et avril 2009. Les paramètres métalliques et physicochimiques étudiés sont le fer, le chrome, la température, le pH, la conductivité électrique, les matières en suspension, la couleur apparente et les ions ammonium. Dans l'ensemble, l'analyse des effluents de la ville de Nouakchott a montré la présence du fer et du chrome à des concentrations variables dont les teneurs maximales atteignent respectivement $542 \mu \mathrm{g} / \mathrm{litre}$ et $368 \mu \mathrm{g} / \mathrm{litre}$. L'application de l'Analyse en composantes principales nous montre que le fer et le chrome sont contenus dans les effluents industriels.

(C) 2010 International Formulae Group. All rights reserved.

Mots clés : Métaux, Idini, Moughataa, Sebkha, Mauritanie.

\section{INTRODUCTION}

Les métaux lourds que l'on trouve dans les eaux usées urbaines ont des origines multiples : ils proviennent des produits consommés au sens large par la population, de la corrosion des matériaux des réseaux de distribution d'eau et d'assainissement, des activités de service (santé, automobile) et éventuellement de rejets industriels (Cauchi et al., 1996).

La présence du fer est attribuable aux eaux acides de drainage des mines, aux eaux de lessivage de décharges contrôlées, aux effluents d'égouts ainsi qu'aux rejets des secteurs industriels qui traitent le fer. Les eaux usées peuvent être chargées en fer du fait des phénomènes de corrosion dans les conduites ou du matériel de fabrication (Hem, 1972; Olivier et al., 1975). La présence du chrome est le plus souvent liée aux rejets industriels (ateliers de galvanoplastie, de tannage, etc.) (Sepher et al., 2005).

Dans le but de suivre l'évolution du fer et du chrome contenus dans les effluents de la ville de Nouakchott et de caractériser ces deux métaux, nous avons utilisé une méthode 
statistique appelée Analyse en Composantes Principales (ACP), couplée à quelques paramètres physicochimiques. L'ACP est un outil d'analyse de données qui permet d'expliquer la structure des corrélations ou des covariances en utilisant des combinaisons linéaires des données originelles. Son utilisation permet de réduire et d'interpréter les données sur un espace réduit (Maliki, 2000). L'ACP a pour objectif de présenter, sous une forme graphique, le maximum de l'information contenue dans une table de données, basées sur le principe de double projection sur les axes factoriels (Lagarde, 1995).

\section{MATERIEL ET METHODES \\ Milieu d'étude}

Nouakchott est une ville portuaire située sur la côte atlantique au niveau de la nappe de Trarza. La nappe de Trarza s'étend le long de la côte atlantique de Nouadhibou au sud du Sénégal. Nouakchott est alimentée en eau potable par le champ captant d'Idini, situé sur la route de l'espoir à environ $60 \mathrm{~km}$ de la ville de Nouakchott. Le climat y est généralement sec toute l'année, avec des pluies faibles et très irrégulières durant l'été. Les températures oscillent entre $28,4{ }^{\circ} \mathrm{C}$ et $36,4{ }^{\circ} \mathrm{C}$ pour les maxima et $14,6{ }^{\circ} \mathrm{C}$ et $25,7{ }^{\circ} \mathrm{C}$ pour les minima. La ville de Nouakchott est une ville côtière (superficie de $400 \mathrm{~km}^{2}$ ) située vers $18^{\circ} 07$ de latitude nord et $16^{\circ} 01$ de longitude ouest, et présente la partie la plus régulière et la plus monotone du littoral mauritanien, aride et ventée (Figure 1). Nouakchott, géographiquement, est ouverte sur la façade atlantique dans un milieu naturel vulnérable et, comme la majorité des villes situées en bordure de la mer, échappent difficilement à des risques littoraux; mais ils se trouvent exacerbés dans le cas de Nouakchott car elle s'étend sur une zone de dépressions argilosalées avec une nappe phréatique subaffleurante et d'anciennes dunes rouges mobiles. Ces dépressions sont séparées de l'océan par un cordon littoral sableux, bas et étroit (Mint El Bezeid, 2006).

\section{Site d'étude}

La Station de Traitement des Eaux Polluées (STEP), a été dimensionnée pour un débit moyen de référence de $2000 \mathrm{~m}^{3} /$ jour, alors que le débit actuellement traité par la station est de $458 \mathrm{~m}^{3} /$ jour estimé à partir des mesures disponibles auprès du service d'exploitation de la Société Nationale De l'Eau (SNDE). Le réseau fonctionnel est d'une longueur de $38 \mathrm{~km}$ environ, desservant le centre ville et la Moughataa de Tevragh Zeina (environ 1800 ménages). Nouakchott ne bénéficie qu'à hauteur de $4 \%$ du système d'évacuation des eaux usées par le biais des égouts (STUDI, 2000).

Les problèmes d'exploitation ont conduit à une dégradation continuelle des capacités épuratoires, jusqu'à l'arrêt complet de la station en 1979. Cet arrêt d'épuration n'a pas empêché que l'arrivée des eaux usées, non épurées, continue sur les sites de maraîchage de Sebkha, malgré un certain nombre d'études alertant sur les risques sanitaires (Cissé et Tanner, 2000). On a choisi comme site du prélèvement la STEP afin d'obtenir une mesure représentative sur l'ensemble des eaux usées drainées par certains quartiers de la ville de Nouakchott et de quelques unités industrielles connectées aussi à la STEP (une Société de mise en bouteille et une société de pêche).

\section{Méthodes d'analyses}

Les prélèvements ont été effectués hebdomadairement au niveau de la STEP au cours des mois de mars et d'avril 2009. Quelques échantillons ont été prélevés au niveau de la société de mise en bouteille et la société de pêche. Les analyses ont lieu immédiatement après prélèvement, au Laboratoire de Chimie de l'Eau de l'INRSP (Institut National de Recherches en Santé Publique) de Nouakchott.

Le $\mathrm{pH}$ et la température ont été déterminés par un $\mathrm{pH}$-mètre de type Hanna muni d'une sonde mesurant la température. La conductivité a été mesurée par un conductimètre de type Hanna. Les matières en 
suspension sont déterminées par filtration d'un volume d'eau usée sur filtre cellulosique (0,45 microns mètre) selon Rodier (1996). Le fer, le chrome, les ions ammonium et la couleur apparente ont été dosés par un spectrophotomètre UV visible modèle $722 \mathrm{~S}$ Beijing. Le fer est dosé par la méthode colorimétrique à l'orthophénantroline. Le chrome total est dosé par la méthode colorimétrique en présence de diphényle carbazone. La couleur est déterminée par la méthode colorimétrique en présence du platino-cobalt, les ions ammonium par la méthode colorimétrique en présence du réactif Nessler.

L'étude statistique a été basée sur l'ACP. Les matrices de corrélations intermédiaires, les corrélations entre les variables et les axes, et les projections des variables dans l'espace des axes F1et F2 ont été obtenus avec un Logiciel XLSTAT 2010.

\section{RESULTATS}

Evolution des paramètres métalliques et physicochimiques

Les Figures 2 à 9 donnent les résultats de quelques paramètres métalliques et physico-chimiques étudiés au cours du mois de mars et avril 2009. Les prélèvements hebdomadaires ont lieu au cours des mois de mars et d'avril 2009. Les prélèvements ont été effectués le $1^{\mathrm{er}}, 8,15,22$ et 29 mars, et le 5, 12,19 et 26 avril 2009.

\section{Etude statistique \\ Application de l'analyse en composantes principales : cas du fer}

Nous avons effectué premièrement le traitement des données par l'ACP, en utilisant comme variables le fer, la température, le $\mathrm{pH}$, la conductivité, la couleur apparente, les matières en suspension et les ions ammonium et les individus, les 9 prélèvements effectués au cours des mois de mars et d'avril 2009. Le Tableau 1 donne les matrices de corrélations inter-élémentaires; le Tableau 2 donne les corrélations entre les variables et les facteurs, et la Figure 10 donne les projections des variables dans l'espace des axes F1et F2.

\section{Application de l'analyse en composantes principales : cas du chrome}

Nous avons effectué deuxièmement le traitement des données par l'ACP, en utilisant comme variables le chrome, la température, le $\mathrm{pH}$, la conductivité, la couleur apparente, les matières en suspension et des ions ammonium et les individus, les 9 prélèvements effectués au cours du mois de mars et avril 2009. Le Tableau 3 donne les matrices de corrélations inter-élémentaires. Le Tableau 4 donne les corrélations entre les variables et les facteurs. La Figure 11 donne les projections des variables dans l'espace des axes F1et F2.

Tableau 1 : Matrice des corrélations inter-élémentaires.

\begin{tabular}{llllllll}
\hline Variables & Fer & T & pH & CE & MES & Couleur & $\mathbf{N H}_{4}^{+}$ \\
\hline Fer & 1 & & & & & & \\
$\mathrm{~T}$ & 0,731 & 1 & & & & & \\
$\mathrm{pH}$ & 0,130 & $-0,476$ & 1 & & & & \\
$\mathrm{CE}$ & 0,065 & $-0,569$ & 0,990 & 1 & & & \\
MES & $-0,634$ & $-0,011$ & $-0,849$ & $-0,809$ & 1 & & \\
Couleur & 0,034 & $-0,215$ & 0,796 & 0,717 & $-0,628$ & 1 & \\
$\mathrm{NH}_{4}^{+}$ & $-0,952$ & $-0,887$ & 0,043 & 0,137 & 0,469 & $-0,053$ & 1 \\
\hline
\end{tabular}


Tableau 2 : Corrélations entre les variables et les facteurs.

\begin{tabular}{lcc}
\hline & F1 (61,33 \%) & F2 (42 \%) \\
\hline Fer & 0,219 & $-0,953$ \\
$\mathrm{~T}$ & $-0,376$ & $-0,901$ \\
$\mathrm{pH}$ & 0,994 & 0,102 \\
$\mathrm{CE}$ & 0,970 & 0,189 \\
$\mathrm{MES}$ & $-0,890$ & 0,424 \\
Couleur & 0,824 & 0,031 \\
$\mathrm{NH}_{4}^{+}$ & $-0,062$ & 0,996 \\
\hline
\end{tabular}

Tableau 3 : Matrice des corrélations inter-élémentaires.

\begin{tabular}{llllllll}
\hline Variables & $\mathbf{C r}$ & $\mathbf{T}$ & $\mathbf{p H}$ & $\mathbf{C E}$ & $\mathbf{M E S}$ & $\mathbf{C o u l e u r}$ & $\mathbf{N H}_{4}^{+}$ \\
\hline $\mathrm{Cr}$ & 1 & & & & & & \\
$\mathrm{~T}$ & 0,027 & 1 & & & & & \\
$\mathrm{pH}$ & 0,512 & $-0,476$ & 1 & & & & \\
$\mathrm{CE}$ & 0,402 & $-0,569$ & 0,990 & 1 & & & \\
$\mathrm{MES}$ & $-0,390$ & $-0,011$ & $-0,849$ & $-0,809$ & 1 & & \\
Couleur & 0,927 & $-0,215$ & 0,796 & 0,717 & $-0,628$ & 1 & \\
$\mathrm{NH}_{4}^{+}$ & $-0,147$ & $-0,887$ & 0,043 & 0,137 & 0,469 & $-0,053$ & 1 \\
\hline
\end{tabular}

Tableau 4 : Corrélations entre les variables et les facteurs.

\begin{tabular}{lcc}
\hline & F1 (56,64 \%) & F2 (29,98 \%) \\
\hline $\mathrm{Cr}$ & 0,686 & $-0,286$ \\
$\mathrm{~T}$ & $-0,393$ & $-0,918$ \\
$\mathrm{pH}$ & 0,976 & 0,090 \\
$\mathrm{CE}$ & 0,940 & 0,202 \\
$\mathrm{MES}$ & $-0,820$ & 0,386 \\
$\mathrm{Couleur}$ & 0,908 & $-0,132$ \\
$\mathrm{NH}_{4}{ }^{+}$ & $-0,005$ & 0,979 \\
\hline
\end{tabular}

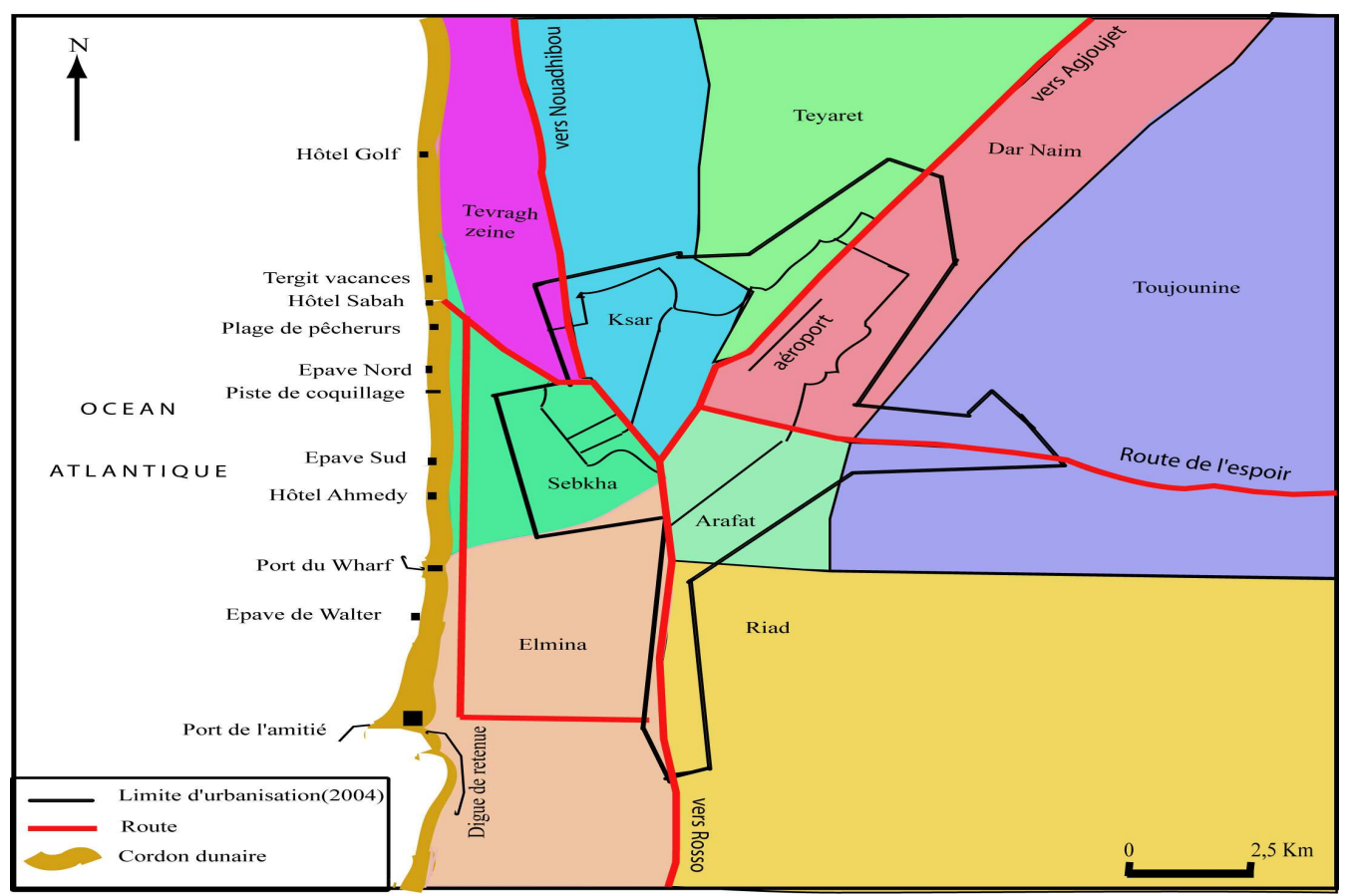

Figure 1 : présentation générale de la ville de Nouakchott. 


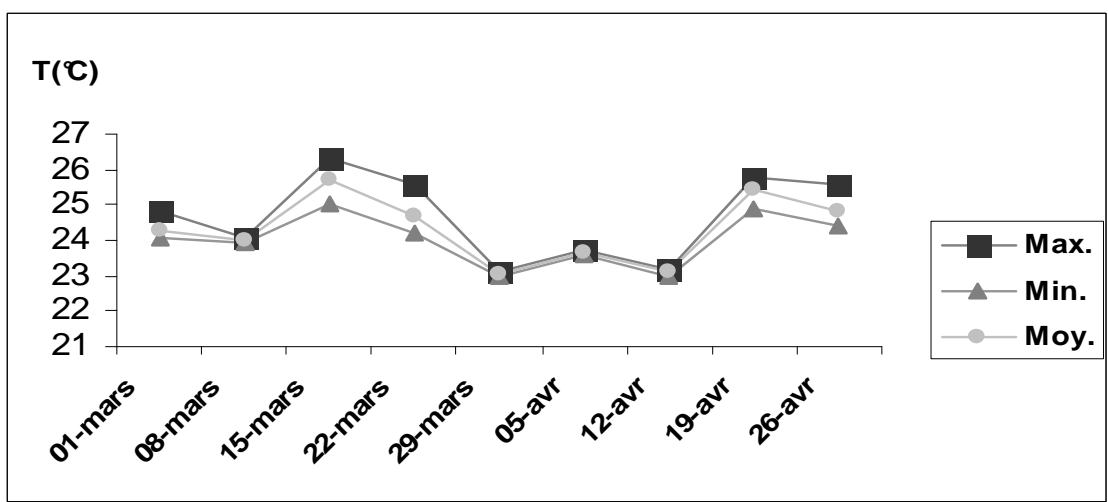

Figure 2 : Evolution de la température des effluents de Nouakchott.

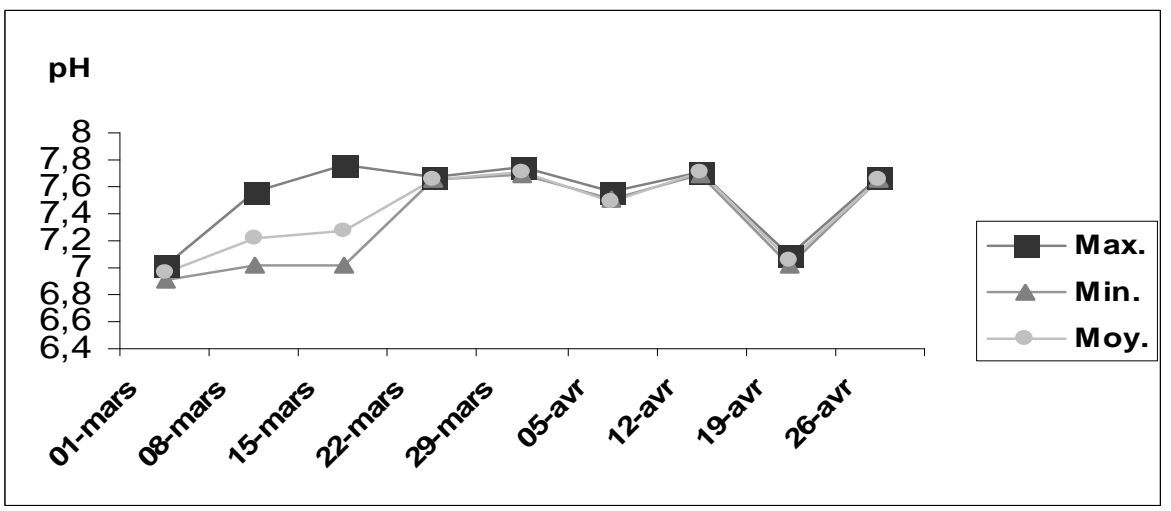

Figure 3 : Evolution du pH des effluents de Nouakchott.

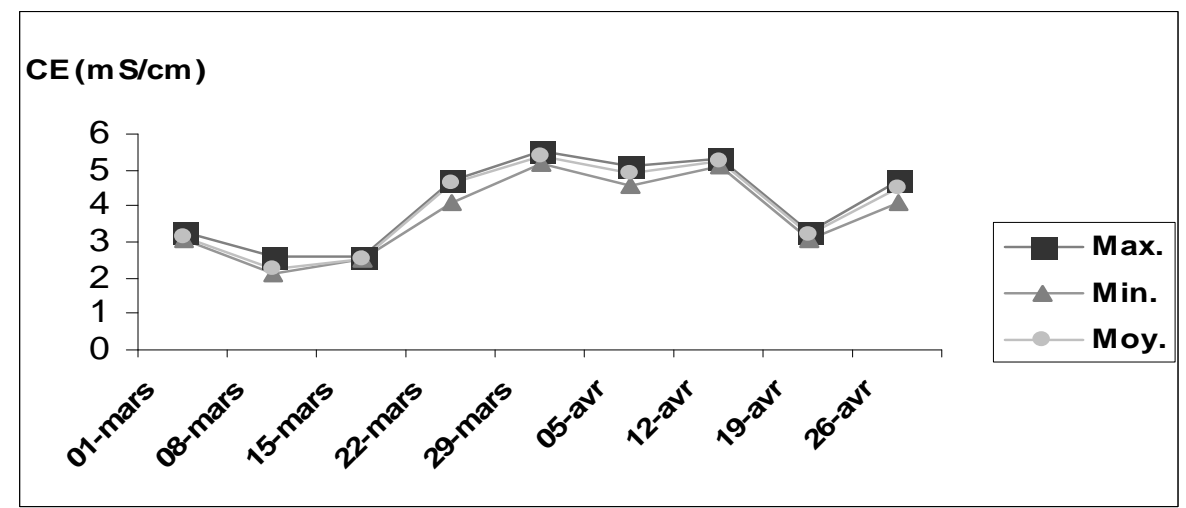

Figure 4: Evolution de la conductivité des effluents de Nouakchott. 


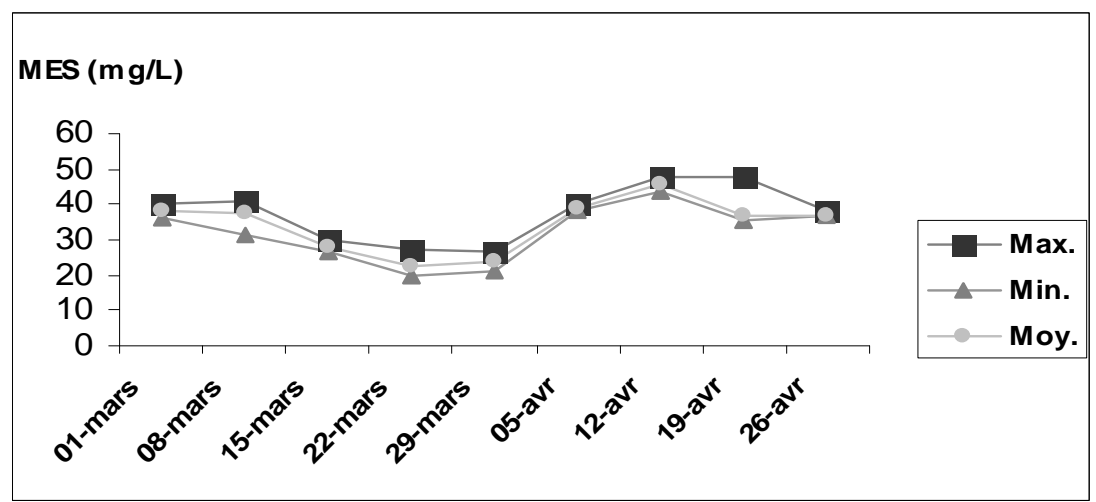

Figure 5: Évolution de la teneur en matières en suspension des effluents de Nouakchott.

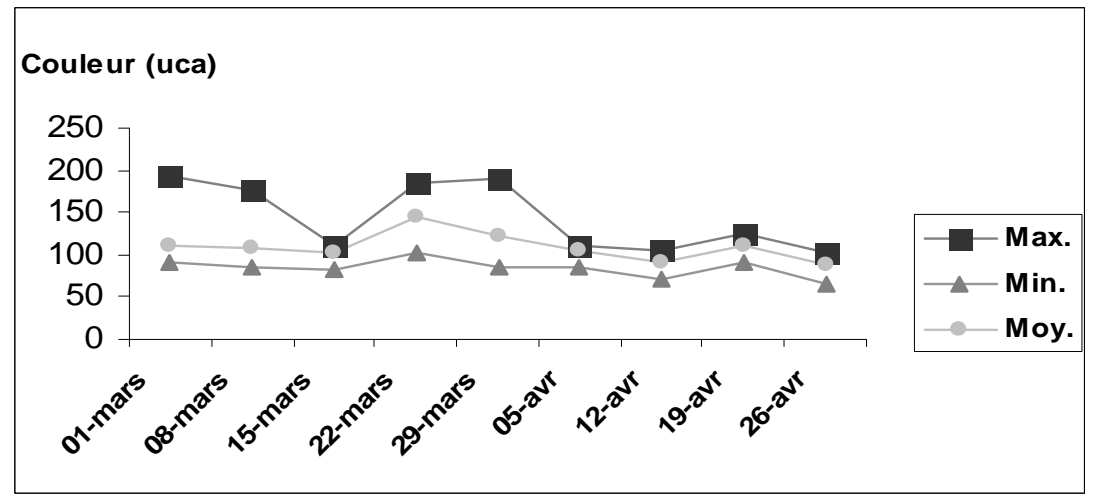

Figure 6 : Évolution de la couleur apparente des effluents de Nouakchott.

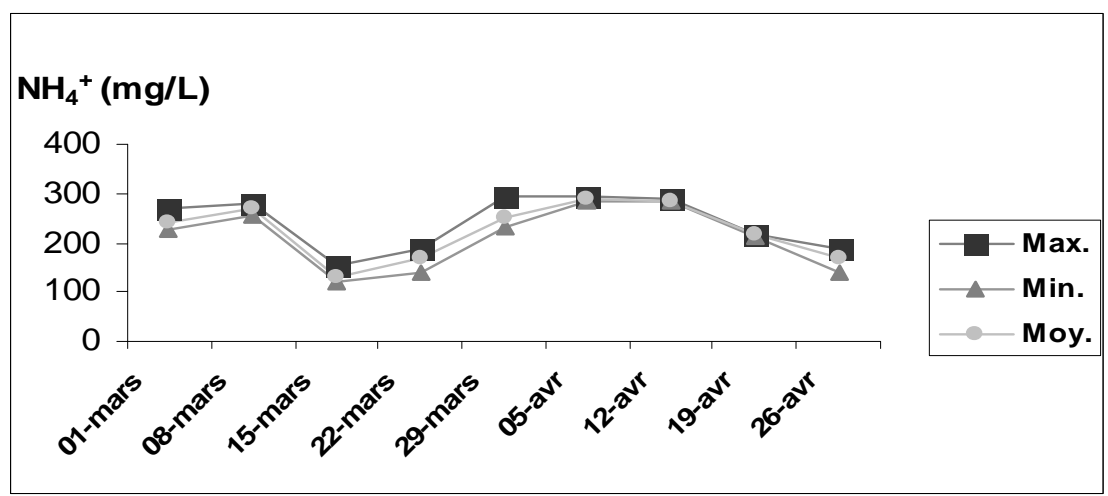

Figure 7: Évolution de la teneur en ions ammonium des effluents de Nouakchott. 


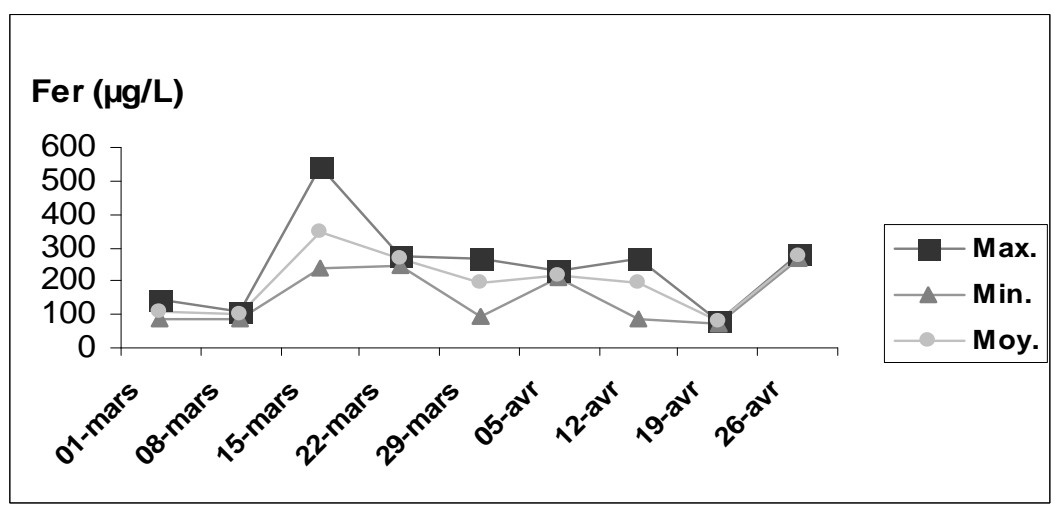

Figure 8: Évolution de la teneur en fer des effluents de Nouakchott.

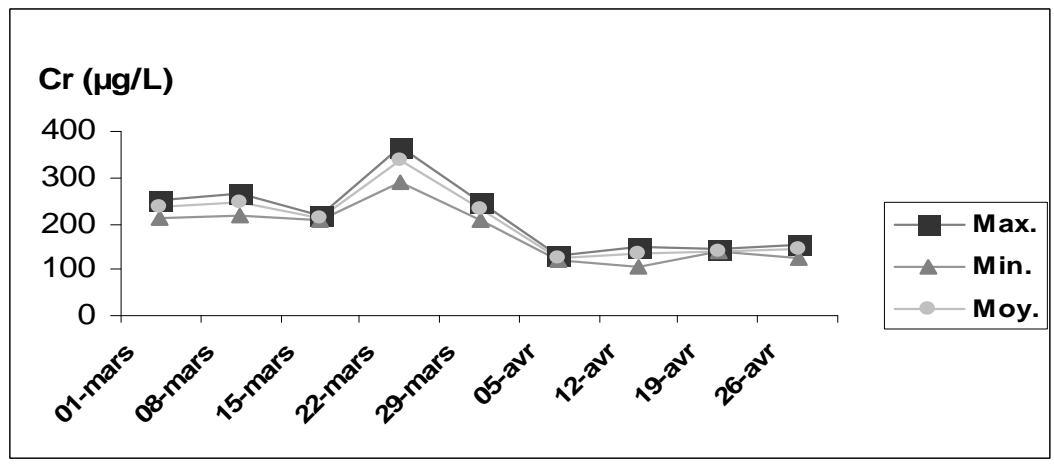

Figure 9 : Évolution de la teneur en chrome des effluents de Nouakchott.

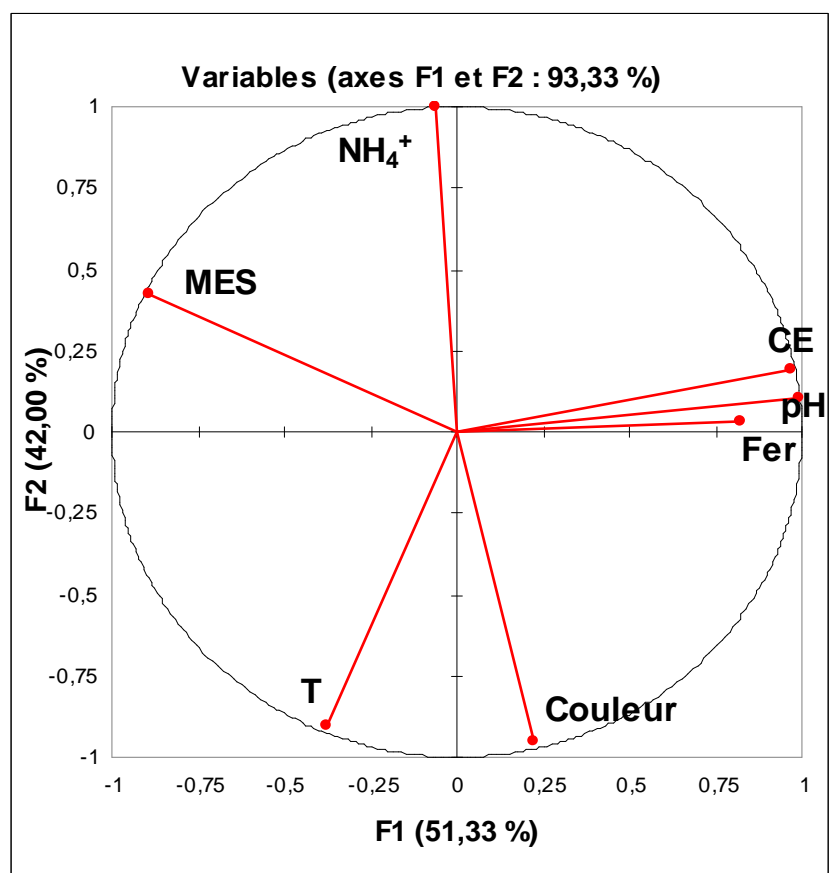

Figure 10 : Projection des variables dans l'espace des axes F1et F2. 


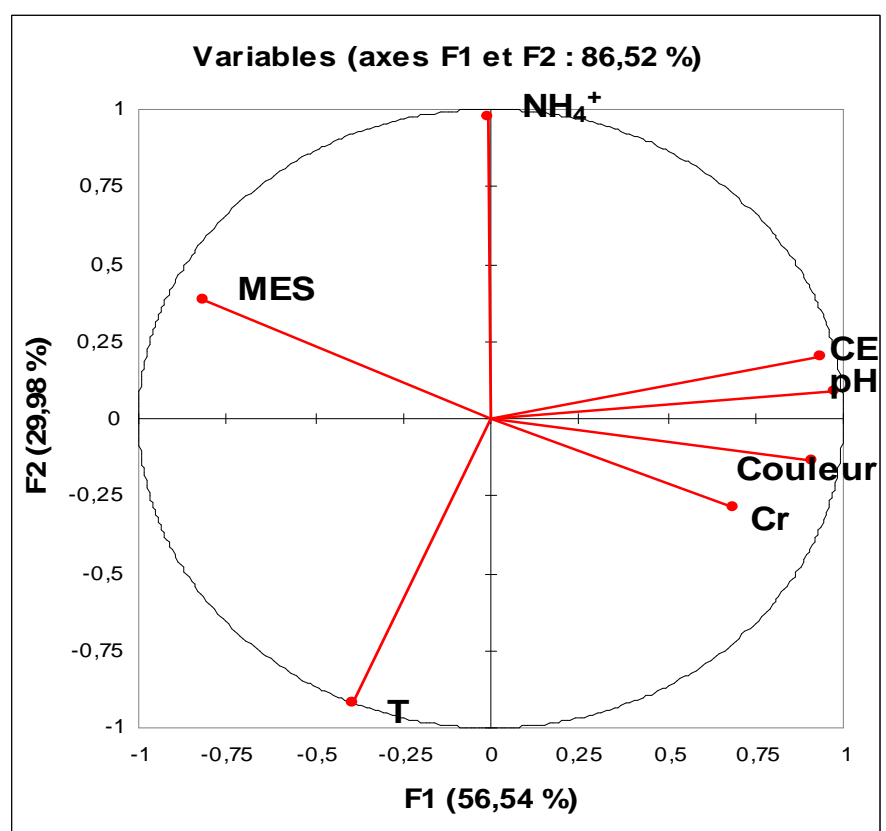

Figure 11 : Projection des variables dans l'espace des axes F1et F2.

\section{DISCUSSION}

Les valeurs moyennes de la température enregistrée au niveau des effluents de la ville de Nouakchott oscillent entre $23{ }^{\circ} \mathrm{C}$ et 25,7 ${ }^{\circ} \mathrm{C}$ (Figure 2). La société de mise en bouteille est équipée de plusieurs machines telles que la chaudière, la soutireuse, la laveuse, etc. Les températures des bains de rinçage des bouteilles ses situent dans un intervalle de 20 ${ }^{\circ} \mathrm{C}$ à $80{ }^{\circ} \mathrm{C}$. L'effluent de la société de mise en bouteille est caractérisé par une température oscillant entre $32,5{ }^{\circ} \mathrm{C}$ et $35,8{ }^{\circ} \mathrm{C}$. Les températures enregistrées au niveau des effluents de la ville de Nouakchott sont inférieures à $35{ }^{\circ} \mathrm{C}$, considérée comme valeur limite de rejet direct dans le milieu récepteur (Ministère de l'Environnement du Maroc, 2002).

Le $\mathrm{pH}$ est un élément important pour l'interprétation de la corrosion dans les canalisations des installations de l'épuration et aussi son effet sur le milieu naturel récepteur. Les valeurs moyennes de $\mathrm{pH}$ des effluents de la ville de Nouakchott varient de 6,9 à 7,7 (Figure 3). Le pH a oscillé entre des valeurs voisines de la neutralité vers des valeurs basiques. Le $\mathrm{pH}$ est un élément important pour l'interprétation de la corrosion dans les canalisations des installations de l'épuration. Au niveau de la société de mise en bouteille la soude caustique est le principal constituant employé pour le nettoyage des bouteilles dont la concentration varie entre 0 et $3 \%$ selon les bains. L'effluent de la société de mise en bouteille est caractérisé par un $\mathrm{pH}$ variant entre 12,13 et 12,24.

La conductivité électrique est probablement l'une des plus simples et des plus importantes pour le contrôle de la qualité des eaux usées. Elle nous renseigne sur le taux de salinité (El Guamri et Belghyti, 2006). Nous observons que la valeur moyenne maximale de la conductivité électrique est de $5,4 \mathrm{mS} / \mathrm{cm}$, et la valeur moyenne minimale de $2,27 \mathrm{mS} / \mathrm{cm}$ (Figure 4). Les valeurs de la conductivité au niveau de la société de mise en bouteille oscillent entre $4,4 \mathrm{mS} / \mathrm{cm}$ et 5 $\mathrm{mS} / \mathrm{cm}$. Les valeurs de la conductivité électrique au niveau de la société de pêche varient entre $29,3 \mathrm{mS} / \mathrm{cm}$ et $30,2 \mathrm{mS} / \mathrm{cm}$. Les 
valeurs de la conductivité enregistrées au niveau des effluents de la ville de Nouakchott dépassent $2 \mathrm{mS} / \mathrm{cm}$ (Journal Officiel de la République Algérienne, 2003).

Les matières en suspension représentent l'ensemble des particules minérales et organiques contenues dans les eaux usées (El Guamri et Belghyti, 2006). Les effluents de la ville de Nouakchott sont caractérisés par une concentration maximale en matière en suspension de $47,33 \mathrm{mg} / \mathrm{litre}$ et une valeur minimale de 22,77 mg/litre (Figure 5). La norme du rejet dans le milieu récepteur en matières en suspension est de $50 \mathrm{mg} /$ litre (Ministère de l'Environnement du Maroc, 2002).

La couleur de l'eau peut être causée par la présence des minéraux comme le fer et le chrome. La couleur apparente est la mesure de la valeur d'une eau qui contient de la matière en suspension. Elle est mesurée sur l'échantillon qui n'a subi aucune filtration ou centrifugation (Black et al., 1963 ; Pemnanen, 1975). La valeur maximale de la couleur apparente au niveau des effluents de la ville de Nouakchott est de 192 uca, et une valeur minimale de 64 uca (Figure 6).

La présence des ions ammonium est à rapprocher de celle des autres éléments azotés identifiés dans l'eau (nitrate, nitrite) et des résultats de l'analyse bactériologique (Rodier, 1996). L'ammonium provient en grande partie des effluents domestiques (urée), etc. (Udert et al., 2003 ; Bonté et al., 2008). La valeur maximale atteinte en ammonium au niveau de l'effluent de la ville de Nouakchott est de $269,29 \mathrm{mg} / \mathrm{litre}$ et la valeur moyenne minimale est de 132,47 mg/litre (Figure 7). La société de mise en bouteille est caractérisée par un effluent contenant des teneurs en ammonium variant entre $0,016 \mathrm{mg} / \mathrm{litre}$ et $1,06 \mathrm{mg} / \mathrm{litre}$, et la société de pêche caractérisée par des teneurs en ammonium oscillant entre 21, $96 \mathrm{mg} /$ litre et 22,80 $\mathrm{mg} / \mathrm{litre}$.

Les effluents de la ville de Nouakchott sont caractérisés par une concentration moyenne en fer de 198,11 $\mu \mathrm{g} /$ litre. La teneur maximale en fer est de $542 \mu \mathrm{g} /$ litre et la teneur minimale de $77 \mu \mathrm{g} /$ litre (Figure 8 ). La société de mise en bouteille est caractérisée par des concentrations supérieures à $0,1 \mathrm{mg} / \mathrm{litre}$ de fer au niveau de l'embouteillage (prélevé dans l'égout rassemblant l'eau de rinçage des machines), la laveuse et du collecteur. Les eaux au niveau de l'embouteillage et de la laveuse montrent des concentrations élevées en fer probablement dues aux conduites en fer des eaux usées et au moment du rinçage aussi. La teneur en fer enregistrée au niveau des effluents de la ville de Nouakchott est très inférieure à $5 \mathrm{mg} /$ litre, considérée comme valeur limite pour les eaux destinées à l'irrigation (Ayers et Wescott, 1985 ; Ministère de l'Environnement du Maroc, 2002).

Les effluents de la ville de Nouakchott sont caractérisés par une concentration moyenne en chrome de 200,44 $\mu \mathrm{g} / \mathrm{litre}$. La teneur maximale en chrome est de $368 \mu \mathrm{g} /$ litre et la teneur minimale de $108 \mu \mathrm{g} /$ litre (Figure 9). L'analyse des échantillons prélevés au niveau de l'effluent de la société de mise en bouteille montre que la teneur maximale du chrome ne dépasse pas $100 \mu \mathrm{g} /$ litre. La teneur en chrome enregistrée au niveau des effluents de la ville de Nouakchott est inférieure à 5 $\mathrm{mg} /$ litre, qui est la valeur limite du rejet du chrome dans le milieu récepteur (Journal Officiel de la République Algérienne, 2003) et aussi inférieure à $1 \mathrm{mg} / \mathrm{litre}$, considérée comme valeur limite à des eaux destinées à l'irrigation (Ministère de l'Environnement du Maroc, 2002).

Une corrélation positive significative a été observée entre le fer et la température, et une corrélation positive faible a été observée entre le fer- $\mathrm{pH}$, le fer - conductivité et le fercouleur apparente (Tableau 1). Une corrélation significativement négative a été observée entre le fer-ammonium et entre le fer-matières en suspension (Tableau 1).

L'analyse du plan factoriel F1 et F2 montre que plus de 93,33\% sont exprimés. L'analyse dans ce plan est donc acceptable (Athamena, 2006). Le facteur F1 représente 
$61,33 \%$ de la variance et il est prédominé par un seul pôle qui est positif. Le pôle positif est formé par le fer, le $\mathrm{pH}$, la conductivité et la couleur (Tableau 2 et Figure 10). Le facteur F2 représente $42 \%$ de la variance et il est prédominé par deux pôles. Le pôle positif est formé des ions ammonium et des matières en suspension. Le pôle négatif est formé de la température (Tableau 2 et Figure 10).

Les travaux de N'diaye et al. (2009) sur la caractérisation physicochimique des eaux usées brutes de la ville de Nouakchott ont montré que les effluents domestiques sont riches en ammonium provenant en général des charges fécales, des urines et des effluents industriels caractérisés par une forte salinité et un $\mathrm{pH}$ élevé. La salinité forte est apportée par la société de pêche et un $\mathrm{pH}$ élevé est apporté par la société de mise en bouteille. Nous pouvons dire que $\mathrm{F} 1$ est un effluent basique ayant une forte salinité, coloré et contenant du fer. F1 est caractéristique d'un rejet industriel. Le fer est apporté par un effluent industriel. Nous pouvons dire probablement que le fer provient de l'effluent industriel.

Une corrélation positive significative entre le chrome et la couleur, une corrélation positive moyennement significative entre le chrome-pH et le chrome-conductivité, et une corrélation positive faible a été observée entre le chrome et la température (Tableau 3). Une corrélation négative moyennement significative entre le chrome et les matières en suspension et une corrélation négative faible a été observée entre le chrome et les ions ammonium (Tableau 3).

L'analyse du plan factoriel F1 et F2 montre que plus de $86,62 \%$ sont exprimés. L'analyse dans ce plan est donc acceptable (Athamena, 2006). Le facteur F1 représente $56,64 \%$ de la variance et il est prédominé par un pôle positif qui est formé par le chrome, le $\mathrm{pH}$, la conductivité et la couleur (Tableau 4) et (Figure 11). Le facteur F2 représente $29,98 \%$ de la variance et il est prédominé par deux pôles, un pôle positif formé par les ions ammonium et les matières en suspension et un pôle négatif formé par la température
(Tableau 4) et (Figure 11). F1 est un effluent basique ayant une forte salinité, coloré et contenant du chrome. F1 est caractéristique d'un rejet industriel. Les paramètres tels que la température, le $\mathrm{pH}$ et la conductivité électrique sont caractéristiques des effluents industriels (N'diaye et al., 2009). Nous pouvons dire probablement que le chrome provient de l'effluent industriel.

\section{Conclusion}

Dans l'ensemble, l'analyse des effluents de la ville de Nouakchott a montré la présence du fer et du chrome à des concentrations variables. Les teneurs maximales du fer et du chrome atteignent respectivement $542 \mu \mathrm{g} / \mathrm{L}$ et $368 \mu \mathrm{g} / \mathrm{L}$.

L'ACP nous montre que le fer et le chrome sont contenus dans les effluents industriels.

\section{REFERENCES}

Athamena M. 2006. Etude des ressources thermales de l'ensemble allochtone su Sétifien, Thèse de Magister, Option : mobilisation des ressources hydriques, Département de l'hydraulique, Faculté des Sciences de l'ingénieur, Université de Batna, $131 \mathrm{p}$.

Ayers R, Westcott D. 1985. Qualité de l'eau en agriculture. Bull. FAO. Irrigation et drainage 29, 80p.

Black AP, Christiman RF. 1963. Characteristics of colored surface water, J. Am. Water. Works. Assoc., 53: 753.

Bonté SL, Pons M, Potier O, Rocklin P. 2008. Relation between conductivity and ion content in urban wastewater. Journal of Water Science, 21(4): 429- 438.

Cauchi, Hyvrard, Nakache, Schwartzbrod, Zagury, Baron, Carré, Courtois, Denis, Dernat, Larbaigt, Dérangére, Martigne et Séguret. 1996. Dossier : la réutilisation des eaux usées après épuration. Techniques, Sciences et Methods, 2: 81118.

Cissé G, Tanner M. 2000. Analyse de la situation de l'agriculture à Nouakchott 
(Mauritanie) et à Ouagadougou (Burkina Faso), Conférence électronique RUAF.

EL Guamri Y, Belghyti D. 2006. Etude de la qualité physicochimique des eaux usées brutes rejetées dans le lac Fouarat, Journal Africain des Sciences de l'Environnement, 1: 53-60.

Hem JD. 1972. Chemical factors that influence the availability of iron and manganese in aqueous systems. Geol. Soc. Am. Spec. Pap., 140.

Journal Officiel de la République Algérienne. 2003. Normes de rejets dans le milieu récepteur, 46, 7-12.

Lagarde J. 1995. Initiation à l'Analyse des Données. Ed. Dunod : Paris; 157.

Maliki AM. 2000. Etude hydrologique hydro chimique et isotopique de la nappe profonde de Sfax (Tunisie). Thèse de Doctorat Fac. Sci. Sfax, 301.

Ministère de l'Environnement du Maroc .2002. « Normes marocaines, Bulletin officiel du Maroc », $\mathrm{N}^{\circ} 5062$ du 30 ramadan 1423. Rabat.

Mint El Bezeid F.2007. Evaluation de risques Environnement qui menacent la zone côtière de Nouakchott et les solutions possibles (Mauritanie). Mémoire DESA, Faculté des Sciences EL Jadida Université Chouaib Doukkali, Maroc.
N'diaye AD, Kankou MOSAO, Sarr AD, Baidy L. 2009. Caractérisation physicochimique des eaux usées brutes de la ville de Nouakchott, Rev. Ivoir. Sci. Technol., 14 : 97 - 109.

Oliver BG, Congreve EG. 1975. Metal concentrations in the sewage, effluents and sludges of some southern Ontario wastewater treatment plants, Environ. Lett. 9: 75.

Pemnanen V. 1975. Humus fractions and their distribution in some lakes in Finland. In Humic Substances, their Structure and Function in the Biosphere, Poveledo D, Golteman HL (éds). Pudoc: Wageningen, Pays- Bas; 207.

Rodier J. 1996. L'analyse de l'Eau Naturelle, Eaux Résiduaires, Eau de Mer ( $8^{\mathrm{m} e}$ édn). Denod : Paris, 1 ; 1383.

Sepher MN, Nasseri S, Assadi MM, Yaghmain K. 2005. Chromium bioremoval from tannery industries effluent by Aspergillus Oryzae, Iran. J. Environ. Health. Sci. Eng., 2: 273-279.

STUDI. 2000. L'assainissement de la ville de Nouakchott : étude d'impacts, $44 \mathrm{p}$.

Udert KM, Larsen TA, Biebow M, Gujer W. 2003. Urea hydrolysis and precipitation dynamics in a urine-collecting system. Water Res., 37: 2571-2582. 